\title{
Image Diagnosis in Extensor Tendons Injuries in the Radiocarpal Region in Horses
}

\author{
Julie Heide Nunes Paz', Daniel de Medeiros Assis', Clédson Calixto de Oliveira², \\ Rodrigo Barbosa Palmeira', Bruno Henrique Rodrigues do Nascimento ${ }^{3}$, Daniela Oliveira ${ }^{4}$, \\ Thiago Arcoverde Maciel $^{3}$ \& Eldinê Gomes de Miranda Neto ${ }^{3}$
}

\begin{abstract}
Background: Soft tissue injuries are common in sport horses, especially those involving tendons, with few current reports in the literature on the diagnosis of extensor injuries, especially with regard to ultrasound characteristics, being essential for the diagnosis of these injuries. The objective of the study is to characterize the clinical signs and the alterations of images, especially ultrasound, in the diagnosis of these lesions of the dorsal radiocarpal region, through case reports of horses seen at the Medical Clinic of Large Animals of the Federal University of Campina Grande (MCLA/UFCG), Patos, PB, Brazil. Cases: The study includes five "vaquejada" competitions horses, of which two were active and the others were away from the sport before the onset of the problem, with injuries to the extensor tendons in the radiocarpal region. Two of the animals had involvement of the common digital extensor muscle tendon (CDEMT), with acute and chronic tenosynovitis, and three with involvement of the extensor carpi radialis muscle tendon (ECRMT), presenting acute tendonitis, septic tenosynovitis and rupture, all of traumatic etiology, except for one whose cause has not been determined. On physical examination, the affected tendon was noticeable in three cases, and two had other injuries associated with the tendons. Bone alteration in the radiographic examination was observed in two cases, however, one of them related to another disease in the radiocarpal palmar region. Ultrasound images, performed in all cases, showed different characteristics and degrees of the involvement of the tendon and its sheath.

Discussion: The scarcity of current reports of extensor tendon injuries in horses can be attributed to the fact that they do not suffer as much overload or effect from biomechanics compared to flexor tendons, in addition to being an area of little soft tissue covering these tendons. Although the involvement of the extensor tendons is more frequent in the pelvic limbs, due to lacerations, the occurrence of lesions in the dorsal radiocarpal region may be related to excess flexion, being easily susceptible to trauma to solid objects. The specific clinical examination, associated with a well-explored anamnesis, can allow us to locate the lesion and achieve a presumptive diagnosis. In three cases, tendon injuries were noticeable on inspection and palpation, important points to guide the diagnosis. However, in two cases with associated injuries this perception became difficult. Regardless of the clinical diagnosis, imaging diagnosis in lesions of the locomotor system was essential, as it allowed us to assess bone involvement, accurately determine the location of the lesion, its extent and prognosis, which would not be possible only in the clinic. Through ultrasonography it was possible to classify the lesions into acute (hypoechoic areas) and chronic (hyperechoic areas or with heterogeneous pattern of variable echogenicity), in addition to the thickness and characteristic of the liquid in the tendon sheath, which are also important aspects for lesion classification. Thus, we conclude that the dorsal region at the carpal level is susceptible to trauma and can easily compromise the extensor tendons. And for the diagnosis and adequate conduct, it is necessary to determine ultrasound image regarding the different types of lesions, which is provided in the present study.
\end{abstract}

Keywords: carpus, tendonitis, tenosynovitis, trauma, ultrasound. 


\section{INTRODUCTION}

Soft tissue injuries are common in sport horses in "vaquejada", usually associated with intense overload or trauma. Those involving tendons occur more frequently, especially in the flexors, with few reports in the literature on injuries of the extensors, especially with regard to ultrasound characteristics. Few studies describe the pattern of normality of these tendons through ultrasound studies of the carpal joint $[5,11,17]$, being even more scarce as the images that characterize the injury patterns.

The carpal and digital extensor muscles occupy the craniolateral portion of the forearm [9]. The tendon of the extensor carpi radialis muscle (ECRMT), the most robust, follows the middle groove at the distal dorsal end of the radius until it is inserted into the metacarpal tuberosity. The tendon of the common digital extensor muscle (CDEMT), immediately lateral to the ECRMT, from its origin, follows dorsolaterally at the level of the radiocarpal region, and dorsally until its final insertion in the distal phalanx. These tendons have the function of extending and fixing the carpal and digital joint, in addition to flexing the elbow joint $[8,14]$. This anatomical and morphofunctional knowledge allows us greater reliability in the interpretation of clinical and ultrasound findings for diagnosis of tendon injuries.

Thus, this article aims to characterize the clinical and ultrasound aspects, seeking to reference the different patterns of injuries to the extensor tendons at the level of the radiocarpal region, through case reports from Medical Clinic of Large Animals (MCLA) of the Federal University of Campina Grande (UFCG).

\section{CASES}

Five reports of "vaquejada" competition horses with tendon injuries to the extensors in the radiocarpal region are summarized in Table 1. Patients 1 and 2 had acute and chronic tenosynovitis, respectively, from CDEMT, while patients 3, 4 and 5, developed tendonitis, septic tenosynovitis and rupture, respectively, of the ECRMT.

Regarding the history, all of them were running "vaquejada", but animals 1, 2 and 4 were away from the sport, days before the problem started. The main complaint of the owners was the increase in volume in the carpal joint and lameness reported in all, except in cases 2 and 5, in which lameness occurred only at the beginning of the problem, including a history of continuous trauma in the stall, in cases 1 and 2. In animals 3 and 5, the clinical signs started after the race, the first one had returned from a competition dragging the limb, improving with the anti-inflammatory treatment instituted in the property, however, he limped again at intermittent periods. Animal 5, performed the function of "esteira" in sport "vaquejada" and had been purchased with a dorsiproximal scar to the carpus, but without any sign of pain. Case 4 had no definite cause.

The animals were raised in an: - intensive system $=$ in bay for $24 \mathrm{~h}$ (case 1$)$; -semi-intensive $=$ in bay during the day and released in the paddock at night (cases 2 and 5); and - extensive system= loose in the pasture (cases 3 and 4).

In the specific physical examination of the locomotor system, the CDEMT was visible and palpable in cases 1 and 2, and very sensitive to palpation (case 1). Patients 3 and 4 had another lesion in the carpal region, in case 4 associated with ECRMT involvement, which was also palpable. In patient 5 , there was an effusion involving the entire dorsal surface of the carpus, but with no evidence of tendon involvement on inspection or palpation. Grade III claudication was evident in patients 1, 3 and 4, and discreet in patient 2 , only after joint flexion for $40 \mathrm{~s}$ followed by guided trot.

After physical examination, the animals were sent to the Diagnostic Imaging Sector. Initially, lateromedial and dorsopalmar radiographic projections of the joint were performed. Only patients 2 (Figure 1) and 3 had bone involvement, although the latter was not related to the extensor lesion, with an area of bone neoformation on the caudal surface of the distal end of the radius. In the lateromedial image of case 2 , it was observed discreet periosteal reaction on the cranial surface of the distal end of the radius. In all cases, the radiographic images showed swelling and slight increase in density in the carpal or radiocarpal dorsal region.

Ultrasonographic examination of the dorsal radiocarpal face was performed in all animals, with a linear transducer of frequency ranging from 8-10 $\mathrm{MHZ}^{1}$. Changes observed in Figure 2A \& B characterize, respectively, acute (case 1) and chronic (case 2) tenosynovitis of the CDEMT. While figure $3 \mathrm{~A} \& \mathrm{~B}$ show, respectively, acute tendonitis (case 3) and septic tenosynovitis (case 4) of ECRMT and Figure 4, rupture of ECRMT (case 5). 
羌帝

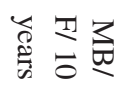

帝

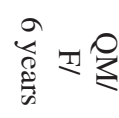

点色

胥.

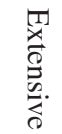

蛋.

竞.

疍

官芯.

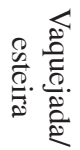

崔

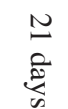

के

F. 可.

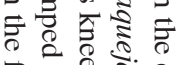

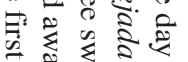

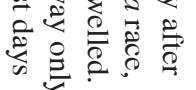

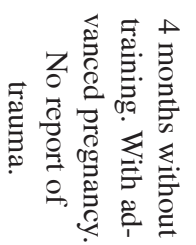

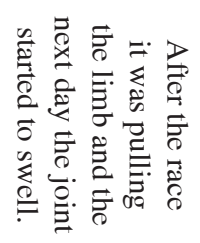

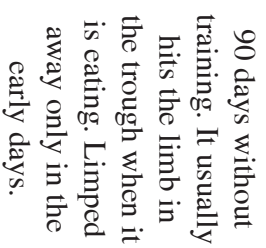

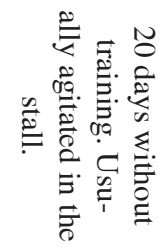

5

줍

5

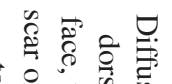

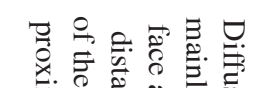

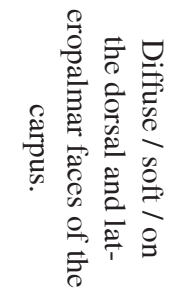

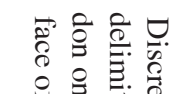

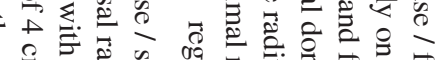

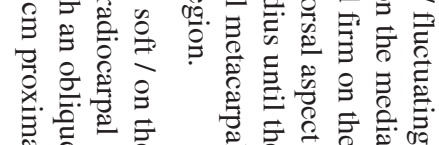

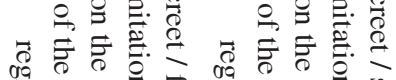

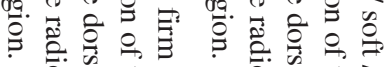

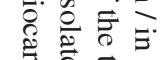

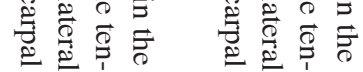

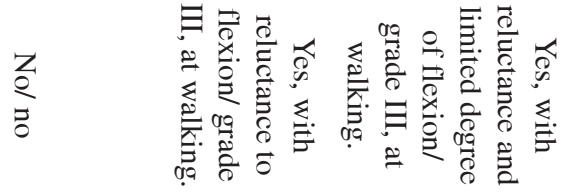

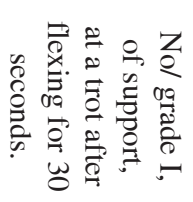

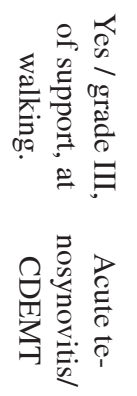

蒙苞

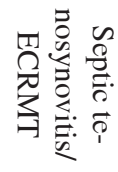

带总害:

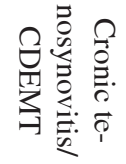

유 우

줍

产

वृ.

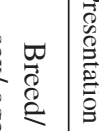

(

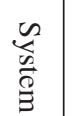

象

突

$\stackrel{n}{\rightleftarrows}$

.

ㄹ.

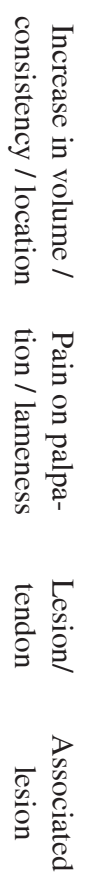

高

$\frac{\overrightarrow{2}}{\frac{2}{5}}$ 政

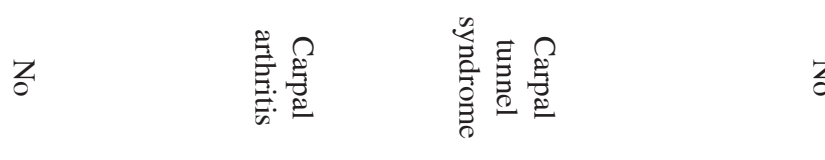

z

z

z

z

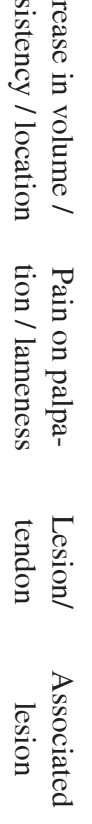

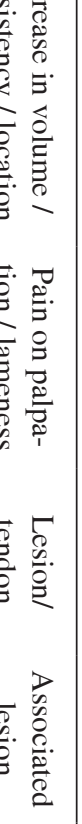

可.

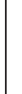

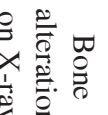

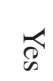

$$
\widehat{\leftrightarrow}
$$

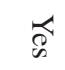

శ্ণ

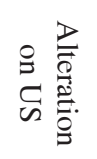


J.H.N. Paz, D.M. Assis, C.C. Oliveira, et al. 2020. Image Diagnosis in Extensor Tendons Injuries in the Radiocarpal Region in Horses.

Acta Scientiae Veterinariae. 48(Suppl 1): 586.

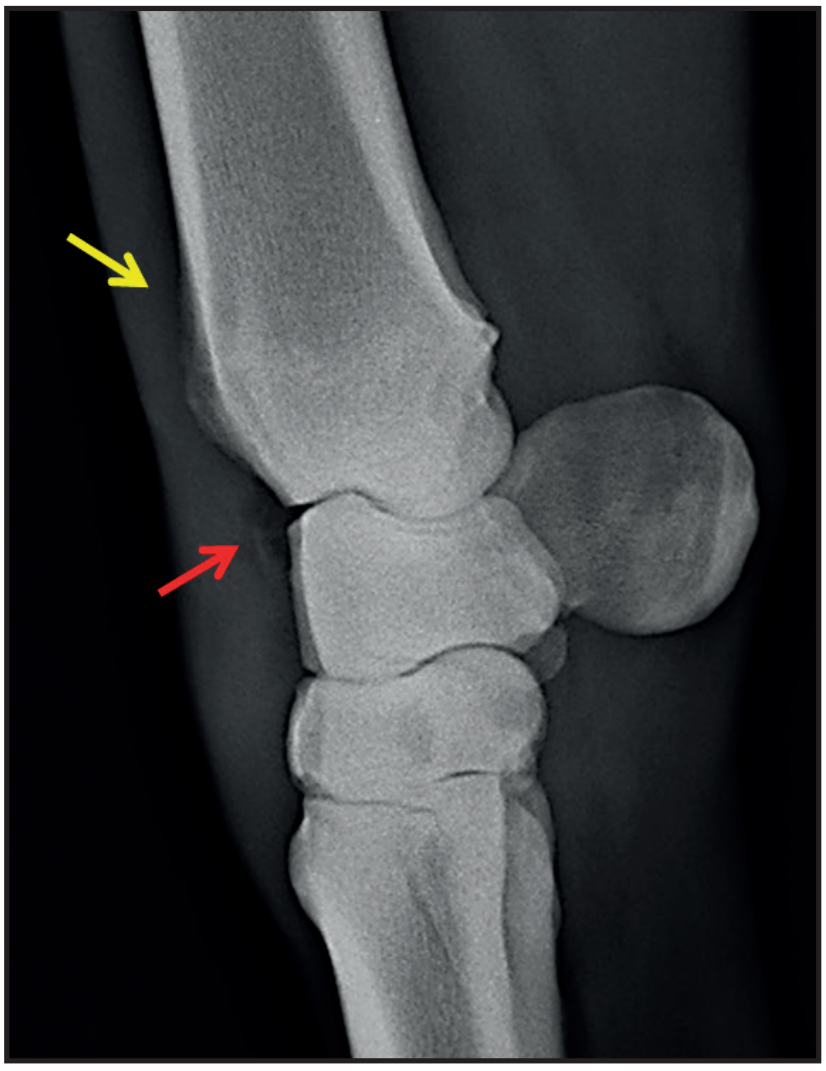

Figure 1. Lateromedial radiography. Case 2: Discreet periosteal reaction on the cranial surface of the distal end of the radius (yellow arrow); radiolucent area in the dorsal soft tissue region of the radiocarpal joint (red arrow); slight increase in density and swelling of the soft tissue on the dorsal face.

In case 4, ultrasound examination also showed thickening of the carpal joint capsule, with increased echogenicity and irregular margins; increase in synovial fluid with a hypoechoic aspect in an anechoic medium and presence of mobile hyperechoic filaments suggestive of fibrin. When associated the images of the tendon and its sheath, with leukogram, which revealed mild leukocytosis with neutrophilia, it directed to the diagnosis of septic tenosynovitis.

\section{DISCUSSION}

The occurrence of injuries to the extensor tendons in horses is currently little reported in the literature, probably because they do not suffer so much overload in biomechanics during athletic activities compared to the flexor tendons. In a retrospective study of horses treated for tendon lacerations, it was observed that $80 \%(12 / 15)$ of animals with extensor tendon involvement returned to activities, higher than the 59\% (13/22) of those who presented flexor injury [7].

Although the involvement of the extensor tendons is more frequent in pelvic limbs (89\%), due to lacerations on the dorsal face of the limb or due to overload [2,16], there are occurrences of septic tenosynovitis resulting from wounds involving the dorsal face of the carpus [3,13]. The first case of CDEMT traumatic tendonitis, resulting from a periarticular osteophyte in the carpal region, evolving to chronic tenosynovitis was reported by Gray et al. [10].

In general, the extensor tendons and their sheaths seem vulnerable to direct damage due to the lack of soft tissue covering these structures in their dorsal aspect. However, the thoracic limbs act as shock absorbers, being more exposed to continuous

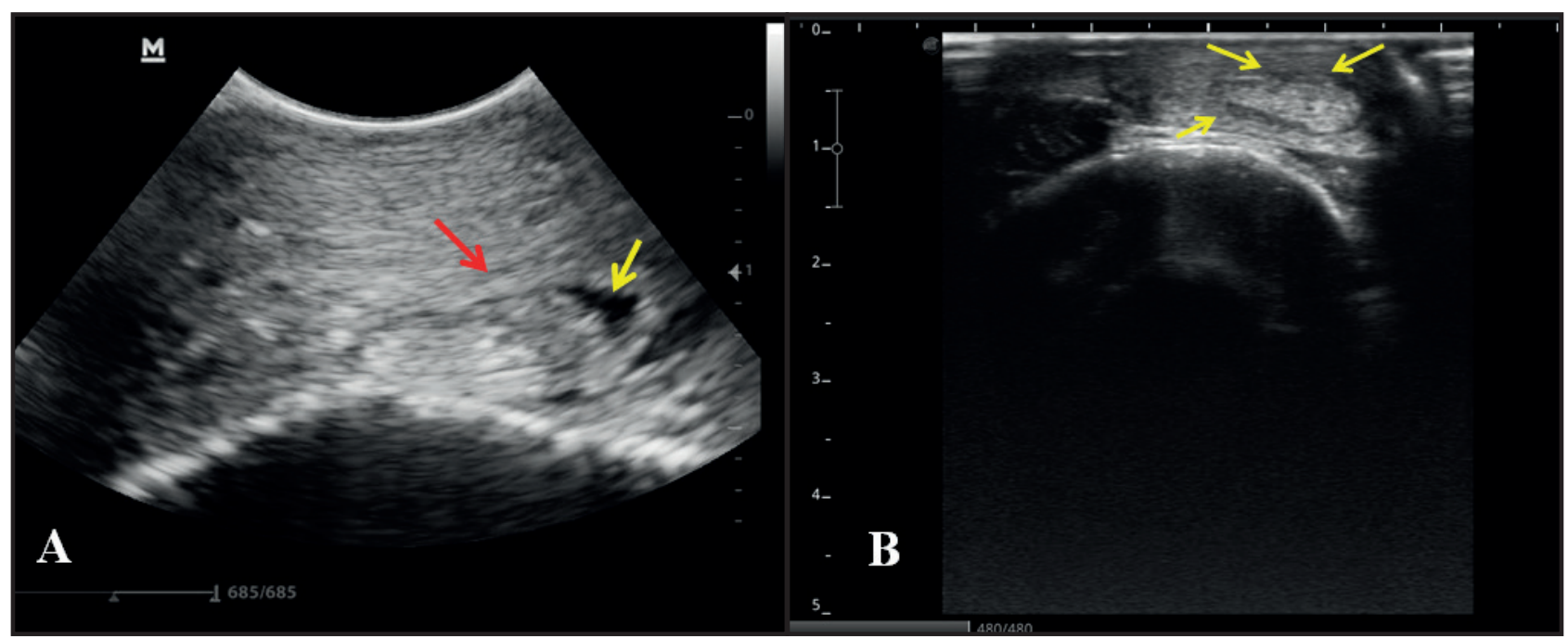

Figure 2. Transverse ultrasound images of the CDEMT in the craniolateral aspect of the distal end of the radius of two horses. A- Case 1: A slight increase in focal synovial fluid (yellow arrow) with an anaechoic characteristic; sheath thickening (red arrow); hypoechoic area extending from the cranial margin of the tendon to the caudal face of the sheath, suggestive of tenosynovitis. B- Case 2: Thickening of the sheath, marked by an evident increase in echogenicity around the tendon; slight amount of hypoechogenic aspect synovial fluid with some points of adhesion between the tendon and the sheath (yellow arrows), and irregularity of its parietal and visceral margins, suggestive of chronic tenosynovitis. 
J.H.N. Paz, D.M. Assis, C.C. Oliveira, et al. 2020. Image Diagnosis in Extensor Tendons Injuries in the Radiocarpal Region in Horses.

tension and constant risk of damage [9]. Its occurrence in the radiocarpal region can be associated with the excess flexion of this joint, being able to easily reach solid objects, as suggested by Wallace [18]. This is consistent with the reported cases 1 and 2, where the stabled animals presented behavior that may have led to traumatic episodes, causing direct and continuous damage to the CDEMT and its sheath.

In cases 3 and 5, the injuries appear to be related to the practice of sport, due to overload or direct trauma. Although case 3 had another disease on the palmar face of that region, the sign presented by the animal shortly after the race suggests involvement of the ECRMT. The cause of the carpal tunnel syndrome was pre-existing, since this pathology is characteristic of chronic injuries [1].

In a study of the pathogenesis of total or partial tendon rupture, Fackelman [6] divides it into two categories: mechanical and degenerative, both related to previous trauma, even though it still not rules out the possibility of the occurrence of rupture is due to intense stress, caused by a direct loss of elasticity of its fibers. However, in patient 5, the scar shown in the proximal region of the carpus and the absence of clinical signs reported before the race suggest that the animal mechanically injured the tendon before it was purchased, through lacerating trauma. When it was exercised again, it developed a recurrent post-traumatic inflammatory process in the same location, showing acute signs of effusion and lameness. This theory becomes more conclusive due to the function of "esteira" exerted by the animal in the "vaquejada", that runs in line with the ox until it is dropped by the "puxada", when it immediately returns, remaining in the front, becoming a propitious moment to trauma in the thoracic members.

Occasionally, when it comes to the tendon, the specific clinical examination of the locomotor system, composed of inspection, palpation and assessment of lameness associated with a well-explored anamnesis, allows us to locate the lesion and presuppose the diagnosis. In cases 1, 2 and 4, the increase in volume delimiting the tendon made it visible and/or palpable, with localized painful sensation (cases 1 and 4), being important starting points to guide the diagnosis. However, regardless of the clinical diagnosis, imaging resources for injuries to the locomotor system are essential. In a complementary way, these resources allowed us to accurately determine the location of the lesion, its extent and prognosis, which would not be possible only in the clinic. It is important that tendon injuries are classified according to time, extent, severity and pathogenesis [6].

The diagnosis of carpal tunnel syndrome, case 3 , occurred due to history, clinical signs, and radiographic alterations, consistent with results described in

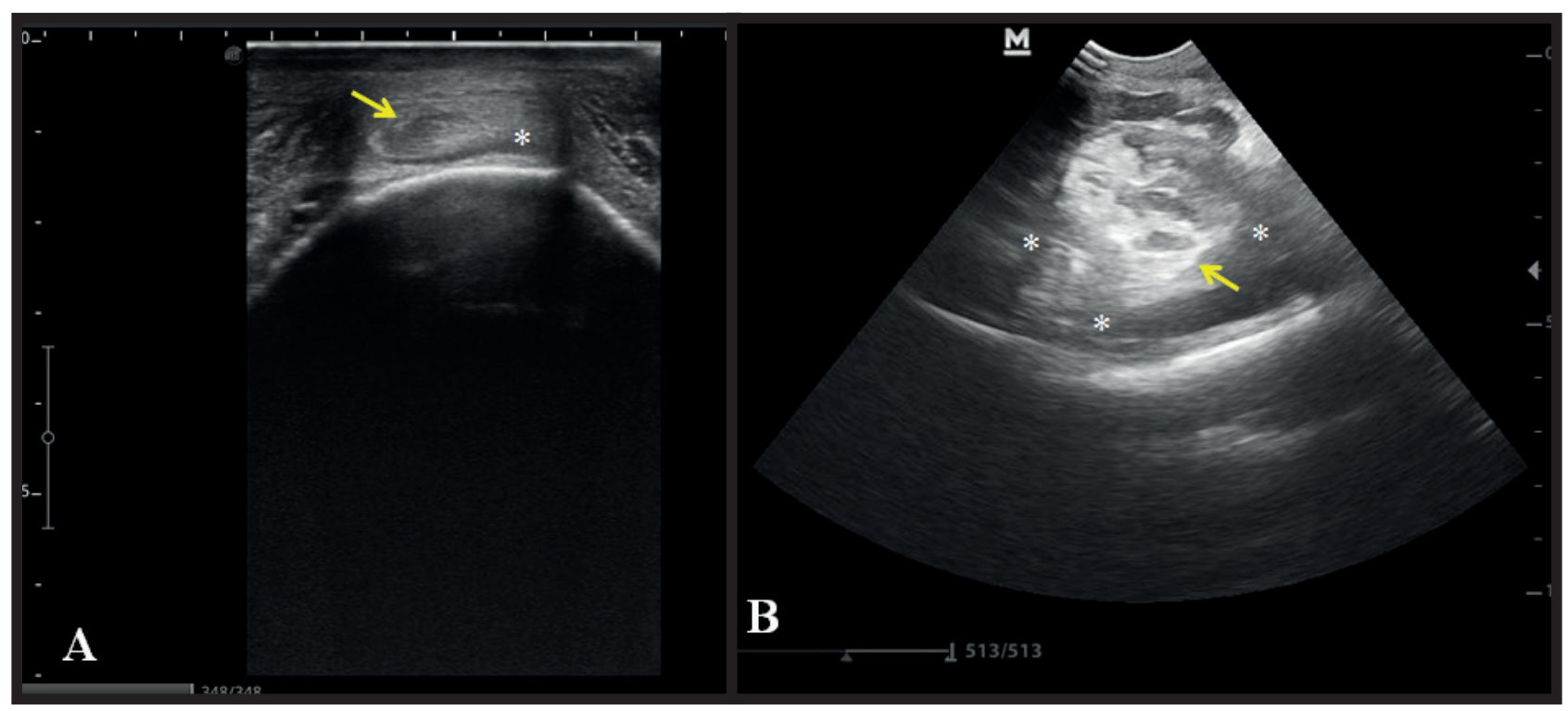

Figure 3. Transverse ultrasound images of the ECRMT in the cranial aspect at the level of the radiocarpal region of two horses. A- Case 3: Hypoechoic lesion in the medial portion of the tendon (yellow arrow); discrete amount of synovial fluid with an anechoic characteristic (asterisk), suggestive of acute tendonitis. B- Case 4: Thickening of the tendon, with a circular aspect, containing hypoechoic areas in almost all its extension and hyperechoic focal area in its caudal margin (yellow arrow); marked degree of hyperechoic synovial effusion in a diffuse hypoechogenic medium (asterisks). Lesions suggestive of tenosynovitis. 
the literature $[1,12]$. These conditions, when there are associated lesions, are often difficult to differentiate in their clinical presentation, demanding a more detailed assessment of the structures surrounding the joint.

Although the suspicion was of a soft tissue lesion, the radiographic image allowed us to assess the existence of associated bone involvement, as a periosteal reaction in the area of passage of tendon structures, observed in case 2 (Figure 1). It was also evidenced radiolucent area in the dorsal region of the radiocarpal joint soft tissue, suggesting effusion of the digital tendon sheath. In all cases, there was a noticeable increase in soft tissue radiopacity on the dorsal radiocarpal face.

In general, in the tendon ultrasound images, the echogenicity, thickness, shape, margins and pattern of the fibers should be evaluated with the transducer in the longitudinal and transverse section $[15,16]$. As a normal ultrasound pattern, in a cross-sectional scan, the ECRMT at the level of the distal region of the radius is described as a homogeneous echogenic structure with an oval shape, becoming more flattened and broad as it approaches the carpus, and elliptical at the level of the radiocarpal region, increasing its volume in the intercarpal portion, and becomes flatter and wider at the level of the carpometacarpal joint. The CDEMT, initially, at the distal end of the radius, is viewed as a triangle and then as an echogenic oval structure, becoming flat as it passes through the groove, already close to the carpus; at the radiocarpal level it narrows slightly and first acquires a concave shape dorsally, later becoming elliptical; in the intercarpal portion, the tendon acquires a more flattened shape, being fusiform at the interface between the third and fourth carpian bones; gradually becoming elliptical and less echogenic as it leans medially under the third metacarpal bone $[5,17]$.

Acute tendon changes are described as hypoechoic areas, similar to those seen in Figure 3A, case 3. On the other hand, chronic lesions are characterized by a heterogeneous pattern of variable hypoechogenicity and hyperechogenicity, as seen in Figure 3B, case 4, although the evident hyperechoic area is small, and when associated with the clinic and time of occurrence suggest injury tending to chronicity [15].

Tendon sheaths are classified according to echogenicity, thickness and amount of internal fluid [16]. Inside, there is usually little or no free liquid, and when present, it is characterized with an anechoic appearance, when in excess, it is a transudate; if this liquid in abundance is hypo or hyperechoic, it represents an increased cellularity or protein content, as in Figure 3B, case 4 , turning a better investigation necessary.

In chronic tendosynovitis an incessant synovial effusion is observed, in addition to fibrous thickening of the sheath, which may be associated with internal stenosis or adhesion between the visceral layer of the sheath and parietal layer of the tendon $[1,18]$. Similar to that observed in Figure 2B, case 2, with the exception of synovial effusion, a condition that may be related to the time of injury, which contributed to intense fibrosis and adherence of the tendon to its sheath. Figure 2A, case 1 , shows a sheath thickening, also characteristic of a chronic injury, however, the time of occurrence, exacerbated signs of pain and the stress behavior in the bay, suggest that the pre-existing injury was exacerbated with the reported trauma.

In a case of CDEMT tenosynovitis of septic nature reported by Booth et al. [4] the ultrasound examination revealed an enlarged tendon, with multiple hypoechoic spots and a marked synovial effusion in the sheath. These changes were similar to those observed in Figure 3B, in case 4, however, in the case of Booth et al. [4] there was a sinus tract between the sheath and the wound, and the confirmation of septic injury was obtained through microbiological analysis of the synovial fluid. In case 4, although the analysis of the synovial fluid was not performed, the ultrasound images associated with the leukogram suggested a septic lesion.

In the longitudinal image, tendon ruptures are characterized by loss of echogenicity and normal fiber alignment, usually represented by an anechoic region, surrounded by an echogenic line, the paratendon. Normally it remains intact unless the injury has occurred by lacerating trauma, in which case there is evidence of damage in the vicinity of the rupture $[15,16]$. This same intact echogenic structure is seen in Figure 4, case 5. Although we have presupposed a percutaneous laceration due to the scar present on the skin, adjacent to the rupture site, the time of occurrence may justify the reconstitution of the paratendon and the absence of clinical signs of pain.

Tendon injuries to the extensors generally have a good prognosis when compared to flexor injuries, even in cases of lacerations. Different functions in 
J.H.N. Paz, D.M. Assis, C.C. Oliveira, et al. 2020. Image Diagnosis in Extensor Tendons Injuries in the Radiocarpal Region in Horses. Acta Scientiae Veterinariae. 48(Suppl 1): 586.

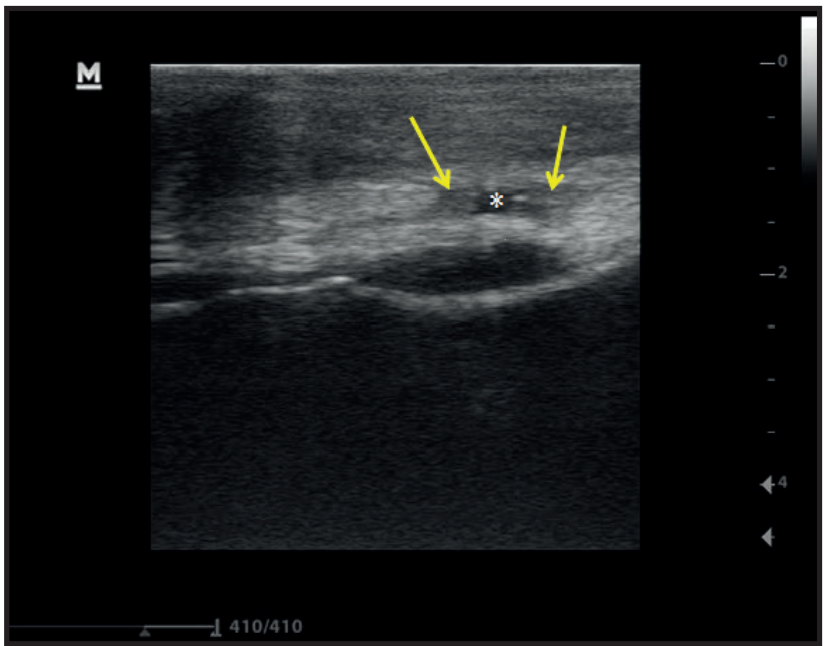

Figure 4. Longitudinal ultrasound image of the ECRMT in the cranial aspect at the level of the radiocarpal region of a horse. Case 5: Loss of continuity of ECRMT fiber parallelism with anaechoic area (asterisk) amid the two stumps of hypoechoic aspect (yellow arrows), characteristic of reorganization of tendon fibers.

biomechanics and weight-bearing are more significant in the flexors, demonstrating greater consequences on sports performance for the horse [19].
The present cases study shows that the occurrence of injuries to the extensor tendons at the level of the carpal region is more related to direct trauma. Few reports have been found in the literature, especially with regard to diagnosis, and not up-to-date. Even more scarce are the references of ultrasound images of these lesions, which proved to be essential for the diagnosis. Finally, it becomes questionable if it is really difficult to occur or if the cases are underestimated due to the low severity that these injuries are able to cause to sport horses.

\section{MANUFACTURER}

${ }^{1}$ Mindray do Brasil - Comércio e Distribuição de Equipamentos Médicos Ltda. São Paulo, SP, Brazil.

Ethical approval. No ethical review or approval from local or national bodies was required as this paper is a case report. The owners of the animals included in this study agreed with the procedures and the use of the information, through the terms of consent generated by the institution.

Declaration of interest. The authors report no conflicts of interest. The authors alone are responsible for the content and writing of the paper.

\section{REFERENCES}

1 Adams O.R. \& Stashak's T.S. 2011. Lameness in Horses. 6th edn. Chichester: Blackwell Publishing, 1280p.

2 Belknap J.K., Baxter G.M. \& Nickels F.A. 1993. Extensor tendon lacerations in horses: 50 cases (1982-1988). Journal of the American Veterinary Medical Association. 203(3): 428-431.

3 Booth T.M., Abbot J., Clements A., Singer E.R. \& Clegg P.D. 2004. Treatment of septic common digital extensor tenosynovitis by complete resection in seven horses. Veterinary Surgeons. 33(2): 107-111.

4 Booth T.M., Clegg P.D., Singer E.R. \& Cheeseman M.T. 2000. Resection of the common digital extensor tendon in a gelding. Veterinary Record. 146(13): 373-376.

5 El-Bably S.H. \& Abdelgalil A.I. 2018. Ultrasonographic Anatomy of the Equine Carpal Region (Equus caballus). International Journal of Veterinary Science. 7(1): 44-49.

6 Fackelman G.E. 1973. The nature of tendon damage and its repair. Equine Veterinary Journal. 5(4): 141-149.

7 Foland J.W., Trotter G.W., Stashak T.S., Mcilwraith C.W., Turner A.S. \& Aanes W.A. 1991. Traumatic injuries involving tendons of the distal limbs in horses: a retrospective study of 55 cases. Equine Veterinary Journal. 23(6): 422-425.

8 Frandson R.D., Wilke W.L. \& Fails A. D. 2011. Anatomia do sistema muscular. In: Anatomia e Fisiologia dos Animais de Fazenda. 7.ed. Rio de Janeiro: Guanabara Koogan, pp.105-147.

9 Gonçalves D.B. 2010. Membro torácico do equino. In: Dyce K.M., Wensing C.J.G. \& Sack W.O. (Eds). Tratado de Anatomia Veterinária. 4.ed. Rio de Janeiro: Elsevier, pp.1155-1228.

10 Gray S.M., Gutierrez-Nibeyro S.D. \& Lobato D.N. 2019. Tenoscopic removal of a protruding osteophyte as a treatment for chronic common digital extensor tendinopathy and associated tenosynovitis. Equine Veterinary Education. 32(7): 73-77.

11 Hage M.C.F.N.S., Invernizzi M.S., Bellegard G.C., Dória R.G.S., Schwarzbach S.V. \& Miada V.J.Y. 2017. Didactic approach of ultrasonographic examination for evaluation of the carpal joint in horses. Ciência Rural. 47(12): 1-6.

12 Nixon A.J. 2002. Arthroscopic Surgery of the Carpal and Digital Tendon Sheaths. Clinical Techniques in Equine Practice. 1(4): 245-256. 
J.H.N. Paz, D.M. Assis, C.C. Oliveira, et al. 2020. Image Diagnosis in Extensor Tendons Injuries in the Radiocarpal Region in Horses. Acta Scientiae Veterinariae. 48(Suppl 1): 586.

13 Platt D. \& Wright I.M. 1997. Chronic tenosynovitis of the carpal extensor tendon sheaths in 15 horses. Equine Veterinary Journal. 29(1): 11-16.

14 Sisson S. 1986. Músculos. In: Getty R. (Ed). Anatomia dos Animais Domésticos. 5.ed. Rio de Janeiro: Interamericana, pp.350-423.

15 Smith R.K.W. 2008. Tendon and Ligament Injury. In: Proceedings of the 54th Annual Convention of the American Association of Equine Practitioners. (San Diego, CA). pp.475-501.

16 Smith R.K.W. \& Cauvin E.R.J. 2014. Ultrasonography of the Metacarpus and Metatarsus. In: Kidd J.A., Lu K.G. \& Frazer M.L. (Eds). Atlas of Equine Ultrasonography. Chichester: John Wiley \& Sons, pp.73-105.

17 Tnibar M., Kaser-Hotz B. \& Auer J.A. 1993. Ultrasonography of the dorsal and lateral aspects of the equine carpus: technique and normal appearance. Veterinary Radiology \& Ultrasound. 34(6): 413-425.

18 Wallace C.E. 1972. Chronic Tendosynovitis of the Extensor Carpi Radialis Tendon in the Horse. Australian Veterinary Journal. 48(11): 585-587.

19 Williams M. 2019. Pathology of equine extensor tendons: Can they function without them? Equine Veterinary Education. 31(6): 315-317. 